\title{
Combined aortic and mitral stenosis in mucopolysaccharidosis type I-S (Ullrich-Scheie syndrome)
}

\author{
T A Fischer, H-A Lehr, U Nixdorff, J Meyer
}

\begin{abstract}
The genetic mucopolysaccharidosis syndromes (MPS) are autosomal recessive inborn errors of metabolism. Heart valve involvement in MPS is not uncommon but only a few case reports of successful cardiac surgery are available. In particular, reports of combined aortic and mitral stenosis associated with MPS type I-S are very rare. Both type I and type VI MPS are associated with significant left sided valvar heart disease that requires surgical valve replacement because of irregular valve thickening, fibrosis, and calcification. A 35 year old man had severe mitral valve stenosis after successful surgical replacement of a stenotic aortic valve. Valvar heart disease was investigated by cardiac ultrasound and left heart catheterisation. Histomorphological characterisation of the affected mitral valve was performed. The case illustrates typically associated clinical features of cardiac and extracardiac abnormalities found in MPS type I-S.

(Heart 1999;81:97-99)
\end{abstract}

Keywords: valvar disease; mucopolysaccharidosis; mitral valve stenosis; aortic valve stenosis

The mucopolysaccharidosis syndromes (MPS) are rare, autosomal, recessive inherited diseases characterised by increased tissue glycosaminoglycan concentrations as a result of decreased enzymatic activity of their degrading enzymes. At least six major entities of this inherited syndrome (MPS types I to VI) have been described, and cardiovascular involvement is known to be occur.

Medicine II, University of Mainz,

Langenbeckstrasse 1, 55101 Mainz, Germany T A Fischer

U Nixdorff

J Meyer

Institute of Pathology, University of Mainz H-A Lehr

Correspondence to: Dr Fischer.

Accepted for publication 29 June 1998 which ultimately revealed the diagnosis of MPS type I-S. The patient is of normal intelligence, and received a high school degree. At age 23 years recurrent syncope resulted in the diagno- sis of severe aortic valve stenosis, which was successfully treated by replacement of the calcified valve with a St Jude Medical prosthesis (St Jude Medical Inc, St Paul, Minnesota, USA).

Ophthalmologic examination because of impaired vision in both eyes revealed cloudy cornea disease. In late 1995 bilateral muscular weakness of the lower extremities developed as well as left sided paraesthesia. Nuclear magnetic resonance tomography of the cervical and thoracic spine showed compression of the spinal cord by severe thickening of the epidural tissue from segments $\mathrm{C} 1$ through $\mathrm{T} 1$. The patient was admitted to the neurosurgery department for surgical decompression of the spinal cord. Preoperative cardiac ultrasound showed an unremarkable, albeit small aortic prosthesis, as well as severe calcific stenosis of the mitral valve. In March 1996, because of increasing neurological symptoms, spinal cord decompression was performed by laminectomy and resection of the epidural tissues. Postoperatively, because of pulmonary congestion, mechanical ventilation was maintained during the early postoperative period and antibiotic treatment was started because of bilateral pneumonia. Positive end expiratory pressure ventilation and diuretic treatment were necessary to control pulmonary oedema. The patient could not be weaned off mechanical ventilation until three weeks postoperatively. His regular sinus rhythm converted to high rate atrial fibrillation, which was controlled with digoxin. Later postoperative outcome was unremarkable and neurological symptoms improved.

$\mathrm{He}$ subsequently underwent right and left heart catheterisation, which confirmed severe mitral stenosis and secondary pulmonary hypertension. In February 1997, after 10 months of outpatient rehabilitation, he was admitted to the cardiothoracic surgical department for replacement of the stenotic mitral valve by a St Jude Medical mechanical prosthesis. Surgery was successful and six months later his cardiopulmonary function had improved remarkably; apart from digoxin and warfarin he required no medication.

Preoperative transthoracic cardiac ultrasound investigation revealed a normal sized, non-hypertrophied left ventricle with regular 

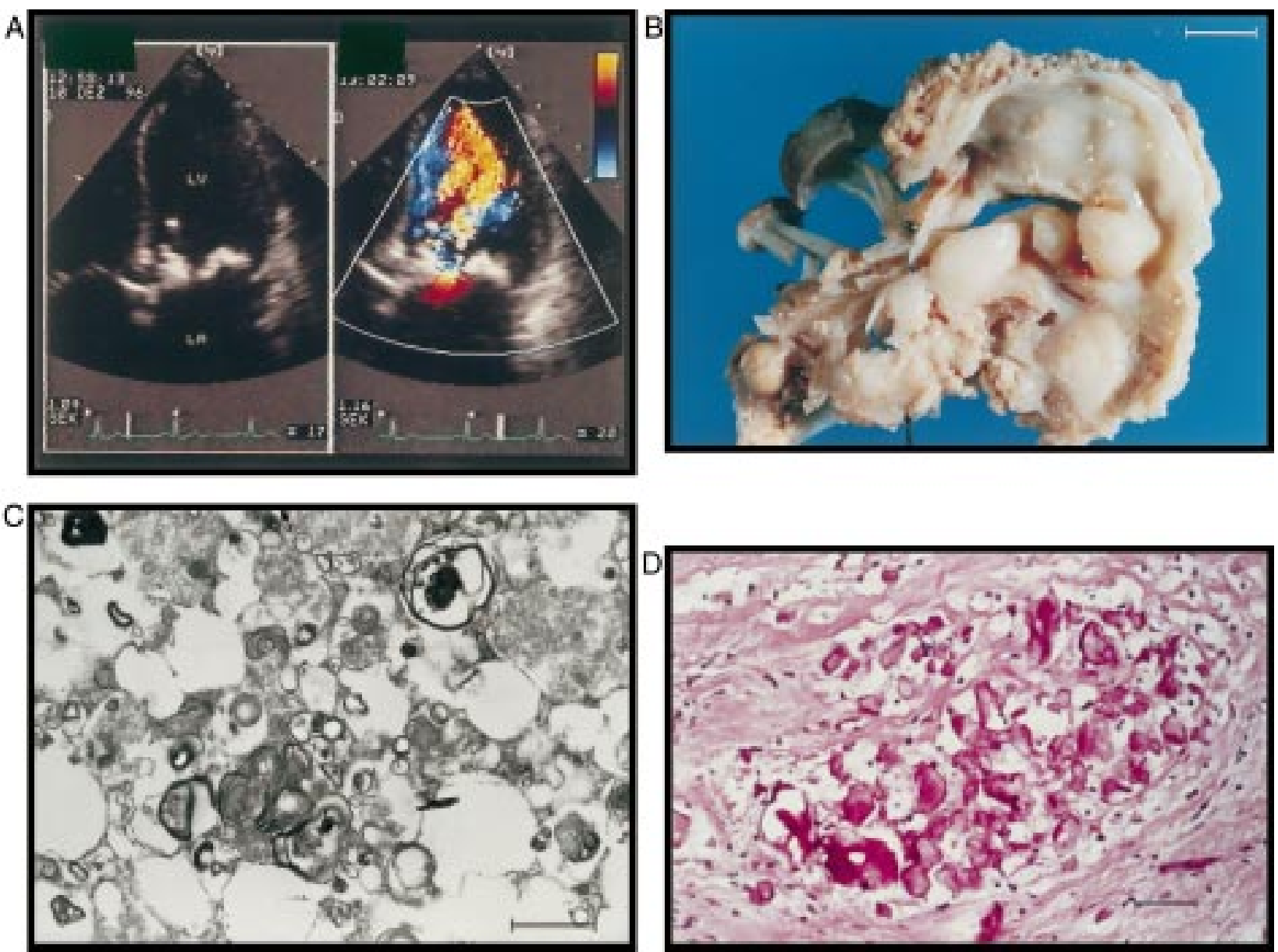

Figure 1 (A) Cross sectional echocardiogram in a right anterior oblique equivalent view (left) and superimposed colour Doppler (right) showing irregular thickening and calcification of the mitral valve and the subvalvar apparatus. Decreased opening of the thickened mitral leaflets led to typical flow acceleration through the stenotic mitral valve. (LV, left ventricle; LA, left atrium.) (B) Excised mitral valve with severe compromise of the central lumen by deposits of mucopolysaccharides. Note the severe thickening of the papillary muscles, chordae tendineae, and the nodular deposition of material along the annulus and leaflets. Some but not all chordae tendineae show severe thickening, calcification, and fusion with neighbouring chordae. (Bar $=1 \mathrm{~cm}$.) (C) Electron micrograph of peroxidase acid Schiff (PAS) positive material depicted in D. Note the vacuolar change of the lysosomes containing fluffy, poorly stained material, and the lamellar structures of lipid leaflets. (Bar $=1 \mu \mathrm{m}$.) (D) Mitral valve leaflet, distended by deposition of PAS positive material and dystrophic calcification. The transverse section through the mitral valve leaflet shows pronounced deposition of irregular masses of PAS positive material resulting in thickening and irregular bulging of the leaflet surface. Also, the leaflet shows extensive dystrophic calcification. $($ Bar $=100 \mu \mathrm{m}$.)

systolic function. Ejection fraction was $60 \%$. The St Jude Medical aortic prosthesis was small with a moderately high systolic gradient of $41 \mathrm{~mm} \mathrm{Hg}$ and a mean systolic gradient of $25 \mathrm{~mm} \mathrm{Hg}$. There was no paravalvar leakage of the aortic prosthesis. Extensive irregular thickening and calcification of the mitral valve apparatus including the chordae was documented (fig 1A). The left atrium was moderately enlarged. The mean diastolic gradient was $10 \mathrm{~mm} \mathrm{Hg}$ with a peak diastolic gradient of $28 \mathrm{~mm} \mathrm{Hg}$. The mitral valve area was $1.2 \mathrm{~cm}^{2}$. There was mild mitral regurgitation. There was secondary pulmonary hypertension with an estimated systolic pulmonary artery pressure of $55 \mathrm{~mm} \mathrm{Hg}$. Diffuse calcification of the mitral annulus including the chordae and the mitral valve leaflets was documented. Motion and function of the aortic prosthesis were unremarkable.

The coronary arteries were free from atherosclerotic lesions. Systolic pulmonary artery pressure was $43 \mathrm{~mm} \mathrm{Hg}$, mean pulmonary artery pressure $31 \mathrm{~mm} \mathrm{Hg}$, cardiac index 3.1 $1 / \mathrm{min} / \mathrm{m}^{2}$, and mean pulmonary wedge pressure
$21 \mathrm{~mm} \mathrm{Hg}$. The pulmonary vascular resistance was moderately increased at 543 dynes.s. $\mathrm{cm}^{-5}$. Mild mitral regurgitation was seen in the left ventriculogram. The cyclic motion of the aortic prothesis was unremarkable. The excised mitral valve was photographed (fig 1B) and sectioned through valve leaflets, annulus, and papillary muscles. Following fixation in buffered formaldehyde, sections were processed for electron microscopy (fig 1C) and routine light microscopy using standard haematoxylin and eosin, alcian blue, and periodic acid Schiff (PAS) stains (fig 1D).

\section{Discussion}

The MPS are a family of hereditary disorders of proteoglycan degrading enzymes. Patients with MPS have intracellular accumulation of acid mucopolysaccharides and glycolipids in various tissues, and excessive urinary excretion of dermatan, chondroitin, and heparan sulphates. ${ }^{1}$ Each type has distinct clinical features and is associated with absent or insufficient activity of specific lysosomal enzymes that degrade the proteoglycans. In MPS type 
I-S (Ullrich-Scheie syndrome ${ }^{2}$ ) the biological defect consists of a loss in enzyme activity of the lysosomal hydrolase-L-iduronidase, ${ }^{3}$ a defect similar to MPS type I-H (Hurler syndrome ${ }^{4}$ ). Thus, acidic glycosaminoglycans accumulate in various tissues, including the heart valves. The most characteristic light microscopic finding is the presence of cells containing large amounts of storage material in the affected organs. These are frequently called "clear cells" as a result of the vacuolisation produced by extraction of the storage material during histological processing, because of their solubility in ordinary fixatives. A characteristic habitus may be less evident than for other types of MPS, but most patients with the UllrichScheie syndrome have characteristic facial features, such as a high forehead and flat nasal bridge. Furthermore, short stature, narrow and rounded shoulders, a short neck, and short stubby fingers have been reported. Corneal opacity with impaired vision is very often associated with MPS type I-H1. In contrast to MPS type I-H severe mental retardation is not typical for type I-S, and intelligence and behaviour have been reported as normal as in types IV (Morquio syndrome) and VI (MaroteauxLamy syndrome). All these features were present in our patient including a remarkable macroglossia and a very narrow throat.

Periarticular soft tissue masses, mainly composed of nodular aggregates of histiocytes, can occur in cartilage and surrounding tissues. ${ }^{5}$ In our patient, similar masses predominately affected the ligaments and dura of the upper spinal cord resulting in local compression of the cord associated with severe pressure induced injury of the myelin. Immediate surgical decompression was needed to prevent irreversible spinal cord damage.

Although cardiac involvement in MPS is not uncommon, only few case reports of successful cardiac surgery are available. ${ }^{6-8}$ In particular, reports of combined aortic and mitral stenosis associated with MPS type I-S are very rare. ${ }^{9}$ Both type I and type VI MPS are associated with significant left sided valvar heart disease that requires surgical valve replacement because of irregular valve thickening, fibrosis, and calcification. In the few cases of MPS type VI undergoing cardiac surgery, both aortic and mitral valves had to be replaced because of dominant stenosis. ${ }^{8}$ In all cases the valve annuli were small so that insertion of adult sized prostheses was difficult.

The increased mortality of patients with these types of MPS is often due to a combina- tion of restrictive lung disease, upper and lower respiratory infections, and advanced valvar heart disease. ${ }^{10}$ Patients with MPS are at high risk from general anaesthesia while attempting endotracheal intubation, and from serious respiratory problems in the early postoperative period. In our patient advanced mitral stenosis was present at the time of the neurosurgical emergency operation and, although the first perioperative hours passed uneventfully, he developed pulmonary oedema (while still in sinus rhythm) and consecutive bilateral pneumonia within the first few days after surgery. Weaning off mechanical ventilation was severely delayed but subsequent outcome was uncomplicated. Thus, the typical anatomy of enlarged tongue and tonsils, mucous membranes, and narrowed lower airway from glycosaminoglycan deposition predispose these patients to respiratory complications. Replacement of the stenotic valve by a mechanical prosthesis was performed a few months later without major complications.

In summary, we describe a very rare case of MPS type I-S (Ullrich-Scheie syndrome) with advanced left sided valvar heart disease with consecutive replacement of a stenotic aortic valve as a young adult and an irregular calcified mitral valve 12 years after the first surgery. Although the syndrome is very rare it might be considered as a possible cause of aortic and mitral valve stenosis in the adolescent and young adult patient.

1 Dorfman A, Matalon R. The mucopolysaccharodoses. In: Stanbury JB, Wyngarden JB, Frederickson DS, eds. The metabolic basis of inherited disease. 3rd ed. New York: metabolic basis of inherited dised

2 Scheie HG, Hambrick GW, Barness LA. A newly recognized form fruste of Hurler's disease (Gargoylism). Am F Ophthalmol 1962;53:753-69.

3 Hopwood JJ, Morris CP. The mucopolysaccharidoses: diagnosis, molecular genetics and treatment. Mol Biol Med 1990;7:381-404.

4 Renteria VG, Ferrans VJ, Roberts WC. The heart in Hurler syndrome. Gross, histologic and ultrastructural observations in five necropsy cases. Am f Cardiol 1976; 38: $487-501$

5 Natowicz MR, Pricilla-Short M, Wang Y, et al. Clinical and biochemical manifestations of hyaluronidase deficiency. $N$ Engl f Med 1996; 335:1029-33.

6 Herd JK, Subramanian S, Robinson H. Type III mucopolysaccharidosis: report of a case with severe mitral valve involvement. F Pediatr 1973; 82:101-4

7 Wilson CS, Mankin HT, Pluth JR. Aortic stenosis and mucopolysaccharidosis. Ann Intern Med 1980; 92:496-8.

8 Tan CTT, Schaff HV, Miller FA, et al. Valvular heart disease in four patients with Maroteaux-Lamy syndrome. Circulation 1992; 85:188-95.

9 Butman SM, Karl L, Copeland JG. Combined aortic and mitral valve replacement in an adult with Scheie's disease. Chest 1989; 96:209-10.

10 Semenza GL, Pyeritz RE. Respiratory complications of mucopolysaccharide storage disorders. Medicine 1988;67: 209-19. 